\title{
COLACIONES HABITUALMENTE CONSUMIDAS POR NIÑOS DE ESCUELAS MUNICIPALIZADAS: MOTIVACIONES PARA SU ELECCIÓN
}

\section{SNACKS USUALLY CONSUMED BY CHILDREN FROM PUBLIC SCHOOLS: MOTIVATIONS FOR THEIR SELECTION}

\author{
Nelly Bustos Z., Juliana Kain B., Bárbara Leyton D., \\ Sonia Olivares C., Fernando Vio del R.
}

Instituto de Nutrición y Tecnología de los Alimentos (INTA), Universidad de Chile, Santiago, Chile.

\begin{abstract}
As part of a school-based intervention to prevent obesity in 7 public schools located in Chile, we carried out a study to determine the children's preferences related to the types of foods purchased at the school kiosk in two of those schools. We interviewed 668,10-13 years old children (53.1\% boys) and in addition to the types of foods purchased, we assessed the foods brought from home. A descriptive analysis of the data was carried out. Sixty percent of the children had money to buy snacks; they mostly bought sweet snacks (35\%), juice and ice cream (33\%) and salty snacks (30\%). Eleven percent of the children bought yogurt and $7 \%$ fruit. Children indicated as motivations to buy high energy snacks: were tasty (82\%), sold at the kiosk (46\%) and are cheap (38\%). It is important to increase the supply of healthy snacks and to develop strategies to motivate school children to prefer them.
\end{abstract}

Key words: school children; snacks; school kiosk; motivations; food purchasing.

Este trabajo fue recibido el 3 de Julio de 2009 y aceptado para ser publicado el 23 de Abril de 2010.

\section{INTRODUCCIÓN}

Desde hace 20 años se viene observando una prevalencia creciente de obesidad, alcanzando cifras preocupantes en la mayoría de los países (1,2). En América Latina la prevalencia de niños con sobrepeso y obesidad sobrepasa el $20 \%$ en más de un tercio de los países (3). Chile no escapa de esta realidad; datos entregados por la Junta Nacional de Auxilio Escolar y Becas (JUNAEB) de alrededor del $70 \%$ de niños de $1^{\circ}$ año básico del país, muestra que la prevalencia de obesidad alcanzó el año 2008 el 20,4\% (P/T > 2 DS, NCHS/OMS) (4). Diversos estudios señalan que las causas principales del aumento de la obesidad en la población infantil son el creciente consumo de alimentos altos en azúcares y grasas y/o la disminución de actividad física (5-7).

En Chile, la información existente sobre las características del consumo de alimentos en los niños reveló que existe un bajo consumo de productos lácteos, verduras, frutas y pescado, y un alto consumo de productos elaborados, ricos en grasas, azúcar y sal en escolares de $3^{\circ}$ a $7^{\circ}$ básico, asistentes a escuelas públicas en tres regiones del país. El 91,5\% de los escolares disponía de dinero para comprar alimentos, entre los que destacaban los bocadillos dulces $(46,5 \%)$ y salados $(42,3 \%)$. Los alimentos que algunos escolares traían del hogar para consumir como colación en la escuela eran similares a los alimentos adquiridos con su dinero (5). En los últimos años se ha observado que los niños, aún los más pobres, disponen de dinero para comprar alimentos. Esto unido a una oferta ilimitada de productos de alta densidad energética y a fuertes campañas de marketing desarrolladas por compañías que comercializan estos productos junto a regalos coleccionables, potencian el consumo de estos alimentos dentro de los colegios $(6,7)$. Olivares y cols. (8) identificaron las barreras para alimentarse en forma saludable que tienen los niños obesos, encontrando que estaban fuertemente asociadas a la publicidad, amplia oferta y bajo costo de las bebidas y alimentos de alta densidad energética, señalando como contrapartida la falta de publicidad, baja disponibilidad y alto costo de los alimentos saludables.

El reciente lanzamiento de la Estrategia Global contra la Obesidad (EGO) por el Ministerio de Salud, representa una oportunidad, que requerirá de la imple- 
mentación y el desarrollo de iniciativas que aborden el problema de la obesidad desde una nueva perspectiva, para lograr mejores resultados (9). Actualmente se está desarrollando una estrategia de educación en alimentación y actividad física para prevenir la obesidad en 1000 escuelas EGO de todo el país (108 en la Región Metropolitana), siguiendo la metodología empleada en el proyecto de Casablanca (10), actualmente en uso en la comuna de Macul.

Aún cuando en el país existen estrategias que promueven estilos de vida saludables al interior de los recintos educacionales (9), no se dispone de modelos validados de intervención que promuevan colaciones saludables durante la jornada escolar. El objetivo de este estudio es mostrar las preferencias alimentarias manifestadas por los niños al comprar la colación en el kiosco del colegio durante el año 2008. Esta información servirá de base para el diseño e implementación de un modelo de intervención que pretende aumentar la oferta de alimentos saludables dentro de los recintos educacionales.

\section{MATERIAL Y MÉTODOS}

Este estudio de corte transversal forma parte de la línea base del proyecto de intervención "Diseño e implementación de un espacio y punto de venta saludable para escuelas básicas de Chile", realizado en mayo del 2008 en dos escuelas básicas municipalizadas de la comuna de Macul (escuelas Villa Macul D-200 y José Bernardo Suárez $N^{\circ} 167$ ). Ambas escuelas fueron seleccionadas por tener un "kiosco" con similar variedad de alimentos, por no disponer de venta de alimentos en la puerta del colegio y por presentar igual índice de vulnerabilidad escolar (IVE) $\geq 60$, medida aproximada de nivel socioeconómico (NSE) que clasificó a los niños en un NSE medio bajo, según criterio definido por la Junta Nacional de Auxilio Escolar y Becas (JUNAEB) (4).

Se incorporó a todos los niños que no participaban en algún programa de control y/o prevención de la obesidad en un centro de salud de la comuna y que no estaban bajo tratamiento nutricional. La muestra estuvo constituida por 668 niños de 10 a 13 años (355 niños y 313 niñas) entre $4^{\circ}$ y $7^{\circ}$ año básico.

Se determinó el origen de las colaciones, la disponibilidad económica de los niños, las preferencias de alimentos en el momento de la compra y las motivaciones de los niños para comprar alimentos de alta densidad energética a través de una encuesta de prácticas alimentarias, cuestionario adaptado del aplicado por Olivares y cols. en el Proyecto FAO/MINEDUC/INTA (5).

\section{Análisis estadístico}

Se realizó una limpieza en la base de datos para detectar inconsistencias y valores fuera de rangos. Una vez hecha la validación se analizaron los resultados de la encuesta en forma descriptiva a través de gráficos y tablas de frecuencias relativas. El análisis fue realizado con el programa estadístico STATA 10 de la Unidad de Estadística del INTA (11).

\section{RESULTADOS \\ Origen de las colaciones}

En la tabla 1 se observa que el $33 \%$ de los niños señaló traer colación sólo desde su casa, el 30\% la compraba sólo en el kiosco y el $29 \%$ señaló que traía colación de su casa y además compraba alimentos en el kiosco. Sólo el 8\% no comía colación.

\section{Alimentos que los niños llevan de colación desde sus hogares}

En la tabla 3 se puede observar que el $62 \%$ de los niños llevaba colación desde sus hogares, de los cuales el $35,6 \%$ y el $34,1 \%$, respectivamente, llevaban fruta y refrescos con azúcar de colación. El 31,1\% llevaba yogur, el $21,5 \%$ productos envasados dulces y emparedados. El $14,2 \%$ llevaba leche, el 12,8\% barras de cereales, $11,3 \%$ bolsitas de cereales y un $10,4 \%$ bebidas gaseosas. Sólo un $2,1 \%$ llevaba frutos secos.

\section{TABLA 1}

Origen de las colaciones en niños de 10 a 13 años de escuelas básicas de Chile $(\mathrm{N}=668)$

Origen de las colaciones

Trae colación de la casa

Compra colación en el kiosco del colegio

Trae colación de la casa y compra colación en el kiosco

No trae ni compra colación
$\%$

33

30

29

8 


\section{Cantidad de dinero del que disponen} los niños para comprar alimentos

El 60\% de los niños declaró que siempre disponía de dinero para comprar alimentos en la escuela. En la figura 1 se observan las cantidades de dinero de las que disponían, cuya mayor frecuencia se concentraba entre los que llevaban \$200 a menos de \$300 (26,7\%); $\$ 300$ a $\$ 400(19,5 \%) ;$ y $\$ 500$ a $\$ 1.000(21,9 \%)$. Un $9,2 \%$ contaba con más de $\$ 1.000$ diarios para comprar alimentos en el kiosco.

El 68\% de los niños señaló que el dinero que utili- zaban para comprar alimentos en el colegio se los daban sus padres, mientras que un 11,8\% indicó a otro familiar como abuelos, hermanos y tíos.

\section{Alimentos que los niños prefieren comprar con su dinero}

En la tabla 2 se puede observar una marcada preferencia por los productos envasados dulces como galletas y chocolates (35\%); los refrescos con azúcar y helados (32\%) y productos envasados salados como ramitas, papas fritas, suflés y galletas saladas (30\%). El 15\% de

\section{FIGURA 1}

Cantidad de dinero que disponen niños de 10 a 13 años de escuelas básicas para comprar alimentos $(\mathrm{N}=668)$

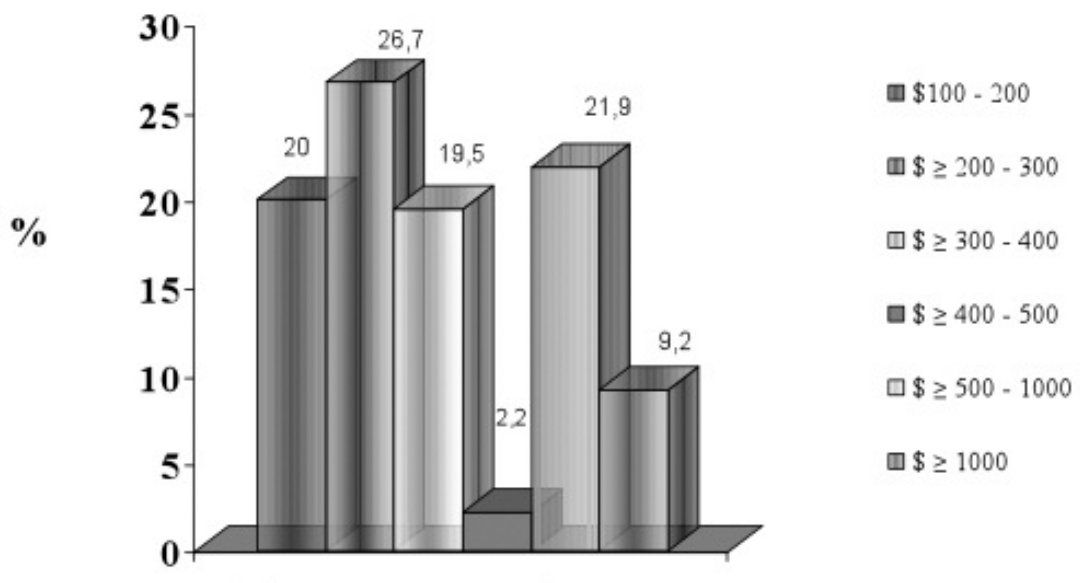

TABLA 2

Alimentos que los niños de 10 a 13 años de escuelas básicas llevan de colación desde sus hogares (N=668)

\section{Alimento}

Jugos

Fruta

Yogur

Productos envasados dulces

Sándwich

Leche

Barra de cereal

Cereales

Bebidas gaseosas

Productos envasados salados

Frutos secos

\section{$\%$}

34.1

36.6

31.1

21.5

21.5

14.2

12.8

11.3

10.4

9.2

2.1 
los niños compraba bebidas gaseosas con azúcar y el $11 \%$ yogur, barras de cereales y hot - dog. Un porcentaje similar (10\%) prefería la leche (cajas individuales) y bolsitas de cereales, un $7 \%$ pizza y frutas y sólo un 5,1\% adquiría frutos secos como maní, almendras o nueces. Un $4 \%$ compraba papas fritas caseras, sopaipillas y sándwich y el $3 \%$ otros productos como chicles y dulces.

Motivaciones de los niños para comer colaciones de alta densidad energética

En la figura 2 se observa que la principal motivación de los niños para comer colaciones de alta densidad ener-

\begin{tabular}{|c|c|}
\hline \multicolumn{2}{|c|}{ TABLA 3} \\
\hline Alimento & $\%$ \\
\hline Productos envasados dulces & 35,0 \\
\hline Jugos & 32,6 \\
\hline Helados & 32,5 \\
\hline Productos envasados salados & 30 \\
\hline Bebidas gaseosas & 15 , \\
\hline Yogur & 11,8 \\
\hline Barra de cereales & 11,5 \\
\hline Completos & 11,4 \\
\hline Leche & 10 \\
\hline Cereales & 10 \\
\hline Pizza & 7 \\
\hline Fruta & 6,9 \\
\hline Frutos secos & 5,1 \\
\hline Papas fritas caseras & 4,5 \\
\hline Sopaipillas & 4,2 \\
\hline Sándwich & 4,2 \\
\hline
\end{tabular}

\section{FIGURA 2}

Motivaciones de los niños de 10 a 13 años de escuelas básicas para comer colaciones poco saludables $(\mathrm{N}=668)$

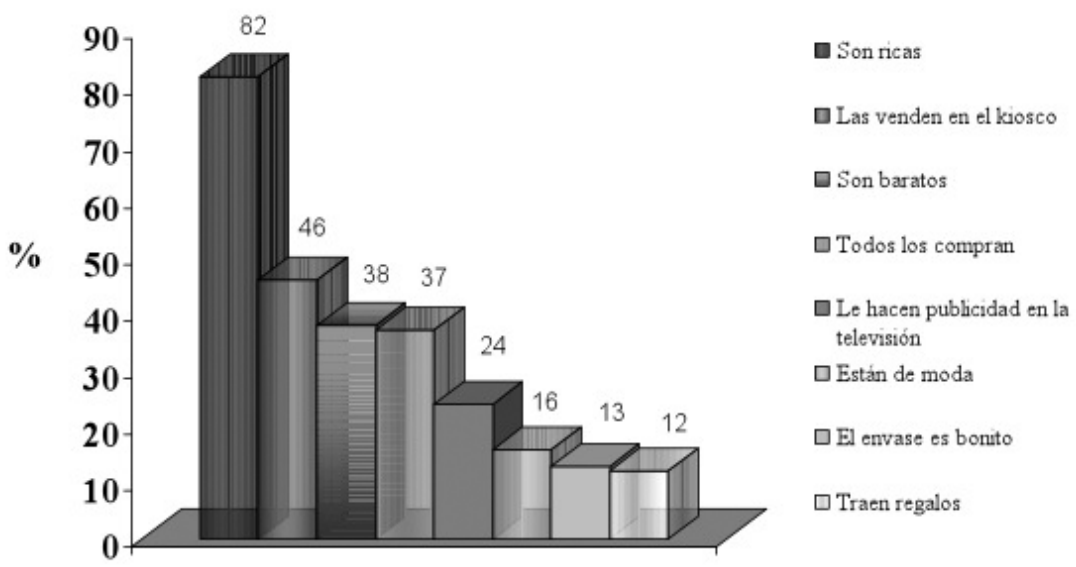

Motivaciones 
gética es el sabor agradable de estos alimentos (82\%), la venta de estos alimentos en el kiosco (46\%) y el bajo precio de estos productos (38\%). El 37\% señaló como motivación el hecho de que todos los niños compren estos productos y el $24 \%$ destacó la publicidad en la televisión. Un 16\% de los niños indicó que los alimentos estén de moda, al 13\% y $12 \%$ los motivaba el envase y la inclusión de regalos en el alimento, respectivamente.

Ante la consulta sobre que los motivaba a comprar alimentos saludables, los niños destacaron que estos alimentos deberían ser más ricos (66\%), más económi$\cos (52.4 \%)$ y que los vendieran en el kiosco $(51.8 \%)$.

\section{DISCUSIÓN}

Gran parte de los niños maneja diariamente dinero para la compra de alimentos en el kiosco del colegio, alcanzando un $60 \%$, porcentaje superior al encontrado por Olivares y cols. el año 2003 en niños de 3 comunas del país, en el que un 34,3\% de ellos siempre disponía de dinero y un $64,2 \%$ sólo "algunas veces" (6).

En relación a los alimentos que los escolares compraban con su dinero, coinciden con los encontrados por Olivares y cols., con un predominio de los altos en grasas, azúcar y sal, como productos envasados dulces, salados y refrescos con azúcar. En el caso del yogur y la leche, éstos alcanzaron el $12 \%$ de las preferencias, porcentaje mayor a lo encontrado por Olivares y cols., con sólo un 6,3\% de las preferencias (6).

Los resultados de este estudio coinciden también con los de Snelling y cols. (12), quienes encontraron que un $83 \%$ de las preferencias en el momento de la compra en las cafeterías de los colegios eran alimentos con alto contenido de grasas, azúcares y sal.

Sólo un 33\% de los niños llevaba colación desde su casa para comer en el colegio, menor a lo encontrado en estudios acerca de la publicidad de los alimentos que alcanzaba un 38,7\% (6). Entre los alimentos que llevaban de colación hay grandes diferencias con estudios anteriores ya que actualmente se observa una tendencia a llevar de colación alimentos saludables como fruta $(36,6 \%)$ y yogur $(31,1 \%)$, mientras que en otros estudios se encontró que el 47,4\% de las colaciones consistía en productos envasados dulces y salados (6).

Entre las motivaciones que los niños de éste y otros estudios han manifestado para comprar alimentos de alta densidad energética, es que estos tienen un precio accesible para ellos, existe una amplia y variada oferta de estos productos dentro del colegio y que tienen buen sabor (8).

Los resultados de este estudio demuestran que es indispensable generar estrategias que tengan como objetivo aumentar la oferta de alimentos saludables dentro de los colegios, acompañadas de campañas de comunicación que motiven a los niños a preferir los alimentos saludables dentro de los recintos educacionales (13-16).

La Organización Mundial de la Salud ha destacado el papel de la escuela como una poderosa cadena para promocionar alimentos a los niños. La preocupación reside en que la mayor parte de los productos ofrecidos, corresponde a alimentos de alta densidad energética y bajo aporte de nutrientes (17). Actualmente en Chile, la Estrategia Global contra la Obesidad, apoyada por la alianza que se ha producido entre las universidades y las empresas de alimentos en el marco de la Responsabilidad Social Empresarial, representan una gran oportunidad para contribuir a hacer más efectivas las intervenciones de promoción y educación que se realizan actualmente, con el fin de frenar la continua tendencia al incremento del sobrepeso, obesidad y factores de riesgo asociados, que actualmente afectan a tantos niños chilenos.

\section{RESUMEN}

Se estudiaron las preferencias de compra de alimentos en el kiosco en escolares de 2 colegios de Chile. Se encuestaron 668 niños entre 10 y 13 años, 355 (53.1\%) de sexo masculino, sobre los alimentos que llevaban de colación, los que compraban con su dinero y las motivaciones para la compra de alimentos. Los datos fueron analizados en forma descriptiva en la Unidad de Estadística del Instituto de Nutrición y Tecnología de los Alimentos (INTA). El 60\% de los niños disponía de dinero para comprar alimentos y los que compraban habitualmente eran productos envasados dulces (35\%), jugos y helados (33\%) y productos envasados salados (30\%). El 11\% compraba yogur y el $7 \%$ fruta. Los niños señalaron como motivaciones para comer alimentos no saludables: son ricos (82\%), los venden en el kiosco $(46 \%)$ y son baratos (38\%). Esto significa que es necesario aumentar la oferta y generar estrategias que motiven a los niños a preferir alimentos saludables dentro de los recintos educacionales.

Palabras clave: escolares; colaciones; kiosco escolar, motivaciones, compra de alimentos.

Dirigir la correspondencia a:

Srta.

Nelly Bustos Zapata

Nutricionista INTA

Universidad de Chile

El Líbano 5524-Macul

Santiago, Chile

Fono: 56-2-9781557

e-mail: nbustos@inta.cl 


\section{BIBLIOGRAFÍA}

1. Organización Mundial de la Salud. Estrategia mundial sobre régimen alimentario, actividad física y salud. 57 Asamblea Mundial de la Salud. WHA57.17. Ginebra: OMS; 2004.

2. Albala C, Vio F, Kain J, Uauy R. Nutrition transition in Chile: determinants and consequences. Public Health Nutr 2002; 5(1A):123-128.

3. Amigo H. Obesidad en el niño en América Latina: situación, criterios de diagnóstico y desafíos. Cad. Saúde Pública, Rio de Janeiro 2003, 19(Sup.1): S163-S170.

4. Ministerio de Educación. Junta Nacional de Auxilio Escolar y Becas. Estado nutricional de escolares hilenos de primer año hásico Santiago, Chile. http://www.junaeb.cl/mapa nutricional. 2009.

5. Olivares S, Kain J, Lera L, Pizarro F, Vio F, Morón C. Nutritional status, food consumption and physical activity among Chilean school children: a descriptive study. Eur J Clin Nutr 2004; 58: 1278-1285.

6. Olivares S, Yañez R, Díaz N, Publicidad de alimentos y conductas alimentarias de escolares de $5^{\circ}$ a $8^{\circ}$ básico. Rev Chil Nutr 2003; 30(1):36-42.

7. McDermott L, Stead M, Hastings G. A marketing strategy to review the effects of food promotion to children. In: G Hastings. Social Marketing. Why should the devil have all the best tunes? London: Elsevier Ltd.; 2007.

8. Olivares S, Bustos N, Moreno X, Lera M, Cortez S, Actitudes y prácticas sobre alimentación y actividad física en niños obesos y sus madres en Santiago,
Chile. Rev Chil Nutr 2006; 33 (2):170-179.

9. Ministerio de Salud. Estrategia Global contra la obesidad EGO-CHILE. www.minsal.cl

10. Kain J, Vio F, Leyton B, Cerda R, Olivares S, Uauy R, Albala C. Estrategia de promoción de la salud en escolares de educación municipalizada de la comuna de Casablanca, Chile. Rev Chil Nutr 2005; 32: 126-132.

11. STATA. Stata 10. Stata Corporation. College Station, USA; 2004.

12. Snelling AM, Korba C, Burkey A. The national school lunch and competitive food offerings and purchasing behaviors of high school students. J Sch Health. 2007; 77: 701-705.

13. Hastings G. Social marketing. Why should the devil have all the best tunes? London: Elsevier Ltd.; 2007.

14. Kotler Ph, Lee N. Social marketing. Influencing behaviors for food. 3rd edition. California: Sage Publications; 2008.

15. Sherry B, McDivitt J, Lipps L, Hans F, Sanders S, Lynn J, Francis L, Scanlon K. Attitudes, practices, and concerns about child feeding and child weight status among socioeconomically diverse white, hispanic, and african-american mothers. J Am Diet Assoc 2004; 104:215-221.

16. Andrien M. Guía metodológica de comunicación social en nutrición. Roma: Organización de las Naciones Unidas para la Agricultura y la Alimentación FAO; 1996.

17. WHO. Marketing of food and non-alcoholic beverages to children. Report of a WHO Forum and Technical Meeting. Oslo, Norway: WHO; 2006. 